\title{
Label-Free and Real-Time Immunodetection of the Avian Influenza A Hemagglutinin Peptide Using a Silicon Field-Effect Transistor Fabricated by a Nickel Self-Aligned Silicide Process
}

\author{
Hyobong Hong ${ }^{1}$, Yang-Kyu Park ${ }^{2}$, Jae-Yeon Kim², Kibong Song ${ }^{1}$ and Chel-Jong Choi ${ }^{2,3, *}$ \\ ${ }^{1}$ Fusion Tech Research Team, Electronics and Telecommunications Research Institute (ETRI), Daejeon 305-700, Korea \\ ${ }^{2}$ School of Semiconductor and Chemical Engineering, Semiconductor Physics Research Center (SPRC), \\ Chonbuk National University, Jeonju 561-756, Korea \\ ${ }^{3}$ Department of BIN Fusion Technology, Chonbuk National University, Jeonju 561-756, Korea
}

\begin{abstract}
Electrical immunodetection of the avian influenza A (H5N1) hemagglutinin (HA) peptide, the IN peptide, with anti-HA antibody was demonstrated using a field-effect transistor (FET) with an n-type silicon ( $\mathrm{Si}$ ) channel and a nickel (Ni) self-aligned silicide source/drain that was fabricated by a conventional top-down process. The specific binding of the IN peptide with anti-HA antibody in phosphate buffered saline (PBS) occurs on the patterned $\mathrm{SiO}_{2}$ surface through covalent linkage. Positive ions in the buffer create majority carriers in the n-type $\mathrm{Si}$ channel, leading to a rapid increase in current across that channel. However, specific binding of the negatively charged antigens on the $\mathrm{SiO}_{2}$ surface overlaying the Si channel results in the reduction of electrons induced in the Si channel by the positive ions, causing a significant decrease in the channel current. The settling time for obtaining a stable signal change, driven by the negatively charged antigens bound to antibody, extrapolates to approximately $32 \mathrm{~s}$. [doi:10.2320/matertrans.M2012068]
\end{abstract}

(Received February 20, 2012; Accepted June 15, 2012; Published August 1, 2012)

Keywords: field-effect transistor (FET), biosensor, avian influenza A, specific binding, antibody, antigen

\section{Introduction}

Influenza A virus is an RNA virus of the family Orthomyxoviridae that infects vertebrates, including human beings. It is usually transmitted though the air or by contact with contaminated material, such as body fluids. ${ }^{1)}$ In recent years, influenza caused by the H1N1 strain has become a global pandemic threat that spreads quickly. Thus, early identification and sensitive surveillance are critical factors for controlling outbreaks. Reverse transcription-polymerase chain reaction (RT-PCR) has become one of the most widely accepted methods to detect and identify the virus. ${ }^{2)}$ However, RT-PCR, or any other PCR-based method, is limited by the usual requirements, such as the correct primer sequences, expensive reagents, and skillful operators. In addition, realtime PCR is the only PCR-based method that does not require additional control reactions to yield quantitative results. Rapid antigen-detection methods, such as immunofluorescence and enzyme immunoassay, can be considered as a complementary approach for the detection of the virus. ${ }^{2)}$ Although these are very simple and effective methods to detect and identify the target virus, they have additional reagent requirements (e.g., the secondary antibodies, and fluorescent and/or other labeling reagents). More recently, a number of signal transduction techniques using immunosensors, based on a quartz crystal microbalance, ${ }^{3)}$ an interferometric biosensor, ${ }^{4)}$ surface plasmon resonance ${ }^{5)}$ or field effect transistor (FET) ${ }^{6}$ ) have been proposed to detect antigen-antibody reactions.

Among these, FET-based biosensors such as genetic FET, ${ }^{7,8)}$ immune FET, ${ }^{9)}$ enzyme FET $^{10)}$ and cell-based FET sensors ${ }^{11,12)}$ have been suggested as a promising alternative to the biochemical labeling methods because of their miniaturization, mass production, and high sensitivity. The fabrication of such biosensors is much more feasible by a top-down

*Corresponding author, E-mail: cjchoi@chonbuk.ac.kr process than by a bottom-up process, and the former is more compatible with the well-established complementary metaloxide-semiconductor (CMOS) process and with monolithic integration, for the construction of electrical interfaces between the biosensor and the signal processing system. Different FET-based biosensors, fabricated by top-down processes, have been successfully employed to demonstrate label-free electrical detection of the HA antigen-antibody: underlap FET, ${ }^{6}$ nanogap embedded FET, ${ }^{6,13)}$ double-gate nanowire FET $^{14)}$ and nanogap double-gate FET. ${ }^{15)}$ However, none of these biosensors are applicable to real-time antigenantibody detection since they all have difficulty in delivering aqueous solution containing target viral antigens to the sensing area, which is required to prevent any leakage current caused by the simultaneous exposure of the solution to the entire sensor surface. In the work described in this paper, we demonstrate label-free and real-time electrical immuodetection of the avian $\mathrm{H} 5 \mathrm{~N} 1$ virus IN peptide using FET biosensors with an n-type $\mathrm{Si}$ channel, fabricated by a conventional top-down process. Importantly, the $\mathrm{Ni}$ selfaligned silicide (salicide) process for forming source/drain regions was employed to fabricate the biosensors, in order to minimize the source/drain underlap length.

\section{Experimental}

\subsection{Fabrication of the Si FET biosensors with Ni- salicided source/drain regions}

Figure 1 shows the schematic diagram of Ni-salicided Si FET biosensors used in this work. The (100) n-type siliconon-insulator (SOI) wafer, with a doping concentration of $1 \times 10^{15} \mathrm{~cm}^{-3}$, was used as a starting material. The thicknesses of the top $\mathrm{Si}$ and the buried oxide (BOX) layers were 100 and $400 \mathrm{~nm}$, respectively. To obtain a thin conducting Si channel with high sensitivity to biomolecules, the thickness of the top Si layer was reduced to $20 \mathrm{~nm}$ by 


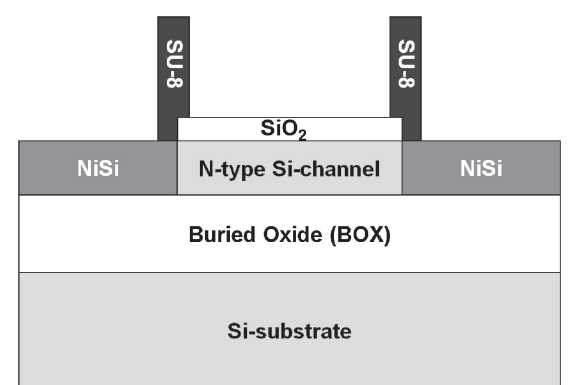

Fig. 1 Schematic diagram of Ni-salicided Si FET biosensors used in this work.

means of a wet thinning process. The Si channel regions, with a width of $16 \mu \mathrm{m}$, were patterned using a dry etching method. For forming the active areas to bind biomolecules, a 6-nm thick $\mathrm{SiO}_{2}$ layer was allowed to grow by a thermal oxidation process, followed by definition of the $40-\mu \mathrm{m}$ length patterns by a photolithography process combined with a wet etching technique. The active areas, where modulation of the $\mathrm{Si}$ channel potential by the targeted biomolecules occurs, were each designed to have a dimension of $16 \times 40 \mu \mathrm{m}^{2}$. For the $\mathrm{Ni}$ salicide process, a 20 -nm-thick $\mathrm{Ni}$ film was deposited by radio frequency magnetron sputtering at room temperature. Then, a rapid thermal annealing (RTA) process was carried out at $400^{\circ} \mathrm{C}$ for $1 \mathrm{~min}$ under a flowing $\mathrm{N}_{2}$ atmosphere. Unreactive $\mathrm{Ni}$ was removed with a mixture of sulfuric acid and hydrogen peroxide. The results of a glancing-angle X-ray diffraction (GXRD) analysis (not shown here) demonstrate that $\mathrm{Ni}$ deposition, followed by the thermal treatment condition employed in this work, results in the formation of an orthorhombic $\mathrm{Ni}$-monosilicide (NiSi) phase with low resistivity. ${ }^{16,17)}$ Finally, the detection reservoir made of the epoxy-based photoresist, SU-8, was formed on active areas. In particular, the SU-8 reservoir was overlapped by active regions (1- $\mu \mathrm{m}$ length) to avoid an electrical short and unintentional change in current, caused by the exposure of solution to the NiSi source/drain regions.

\subsection{Immobilization of the antibodies on the surface of FET sensors}

To immobilize the anti-HA antibody on the Ni-salicided Si-FET biosensor, primary amines were first added to the surface of the patterned $\mathrm{SiO}_{2}$ layer by coating with 3aminopropyltriethoxysilane, followed by subsequent reaction with the cross-linker sulfosuccinimidyl-4[N-maleimidomethyl]cyclohexane-1-carboxylate. This process led to the generation of maleimide moieties on the surface, which in turn react with sulfhydryl groups on antibodies. Ten micrograms of anti-H5N1 HA antibody (ProSci Inc., Poway, CA) in $10 \mu \mathrm{L}$ of PBS buffer $(0.1 \mathrm{M}$ sodium phosphate containing $0.15 \mathrm{M} \mathrm{NaCl}$, with the $\mathrm{pH}$ adjusted to 7.4) was mixed with $490 \mu \mathrm{L}$ of coupling buffer. To this, $50 \mu \mathrm{L}$ of $0.05 \mathrm{M} 2$ mercaptoethylamine (2-MEA) was added to reduce the antibody for coupling, and the mixture was incubated for $90 \mathrm{~min}$ at $37^{\circ} \mathrm{C}$. The reduced antibody was purified on a Zeba desalting column (Thermo Fisher Scientific, Rockford, IL) equilibrated with PBS containing $10 \mathrm{mM}$ ethylenediaminetetraacetic acid (EDTA). The purified antibody was then applied immediately to the sensor surface and incubated for

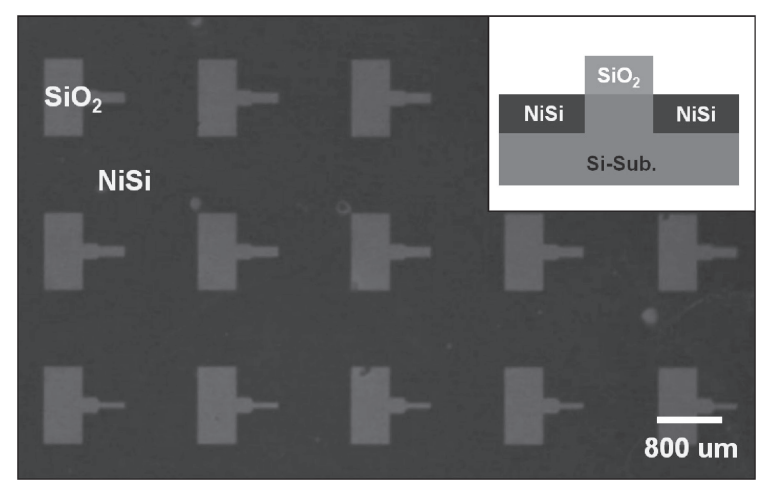

Fig. 2 Confocal fluorescence image taken from a test pattern with the specific binding of antigen-antibody. Inset figure represents the structure of the test pattern.

$4 \mathrm{~h}$ at room temperature. The antibody solution was removed from the biosensor, and its surface was rinsed with coupling buffer. For long-term storage, biosensors were kept in PBS buffer containing $0.02 \%$ sodium azide. Five $\mu \mathrm{L}$ of antigen solution (IN peptide from ProSci Inc., Poway, CA) was diluted in $995 \mu \mathrm{L}$ of PBS. The concentration of diluted solution was $1.0 \mu \mathrm{g} / \mathrm{ml}$. For electrical immunodetection, $1.0 \mu \mathrm{L}(1.0 \mathrm{ng})$ of them are applied to the reservoir of $\mathrm{Ni}$ salicided Si-FET biosensor. To confirm the specific immobilization of the antibody on the surface of $\mathrm{SiO}_{2}$ and the antigen-binding activity of the immobilized antibody, binding of biotinylated-IN peptide was tested. Ten $\mu \mathrm{L}$ of antigen solution was mixed with $10 \mu \mathrm{L}$ of PBS and biotinylated by reaction with $10 \mu \mathrm{L}$ of $10 \mathrm{mM}$ sulfo-NHS-biotin at room temperature for $30 \mathrm{~min}$, and the labeled peptide was purified by passing the solution though a desalting column. After reaction of the biosensor with the biotinylated IN peptide, the bound peptide was reacted with $10 \mu \mathrm{g}$ of fluorescein isothiocyanate (FITC)-conjugated streptavidin (Thermo Fisher Scientific, Rockford, IL, USA) and washed with PBS and water; the bound FITC-streptavidin was then observed by fluorescence microscopy.

\section{Results and Discussion}

Even trace changes in the three-dimensional protein structure and amino acid composition may affect specific binding between antigen and antibody. Thus, prior to demonstrating electrical immunodetection of H5N1 HA with the Ni-salicided Si-FET biosensor, the presence of the antigen-antibody complexes at specific sites on the chip surface was confirmed by confocal fluorescence microscopy. For this examination, the binding of biotinylated-IN peptide to antibody immobilized on the biosensor was visualized by subsequent reaction with FITC-conjugated streptavidin, and the fluorescent patterns of the bound FITC were observed and compared to the test patterns on the biosensor, as shown in Fig. 2. The test patterns were fabricated using the Ni-salicide process described in this work, and its structure is shown in the inset of Fig. 2. The image clearly reveals that antibodyantigen binding occurs only on the patterned $\mathrm{SiO}_{2}$ surface, not on the NiSi surface. This implies that the antigen is selectively bound to the antibody in the correct areas of the biosensor surface, and that the antigen-binding capability 

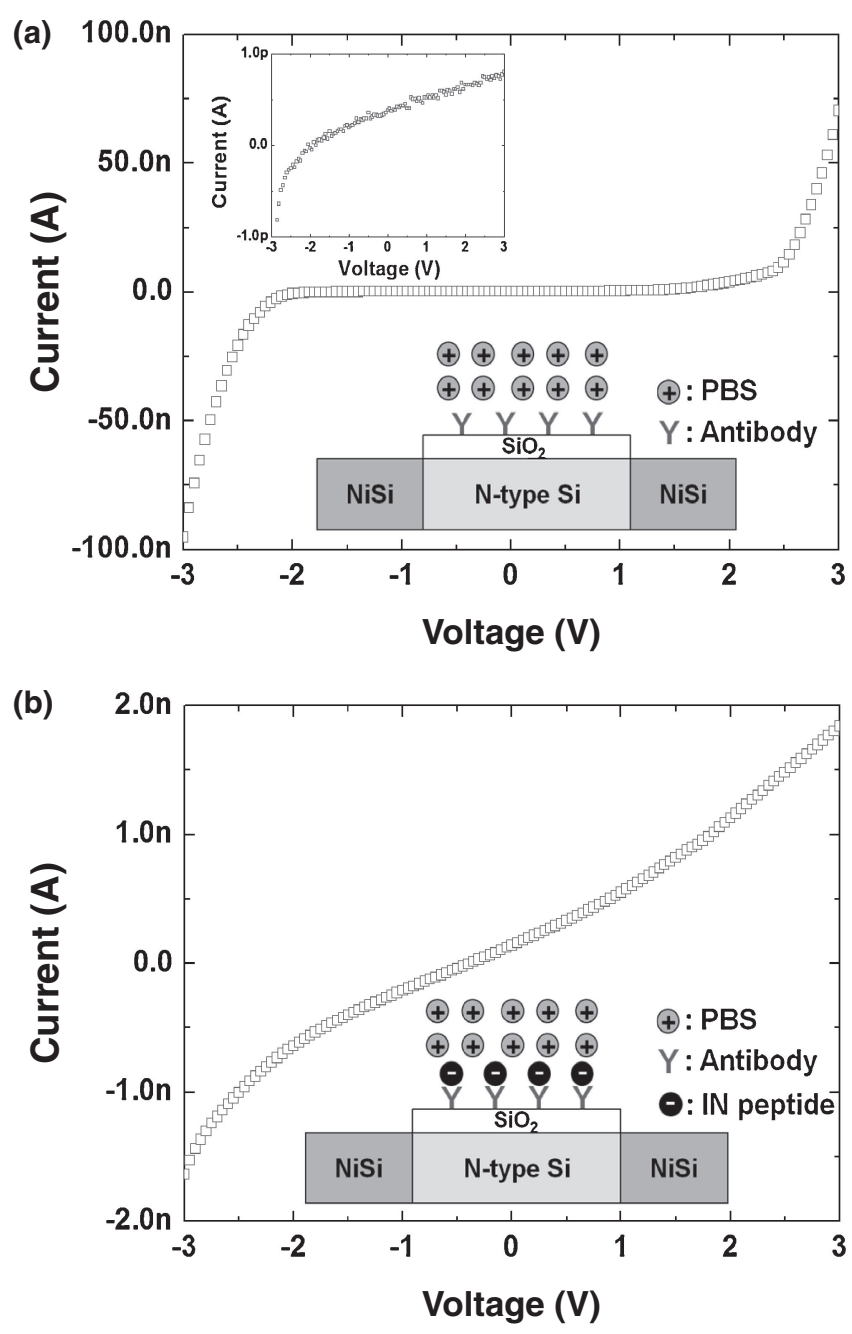

Fig. 3 I-V characteristics of Ni-salicided Si-FET device before and after adding antigen in PBS solution. Note that I-V measurements were begun 2 min after the addition of PBS solutions with and without antigens into the SU-8 reservoir.

of the immobilized antibody remained active during the fabrication and storage conditions employed here.

To investigate the effectiveness of the Ni-salicided Si FET chip as a biosensor for IN peptide detection, electrical immunodetection was carried out in PBS solution with and without peptide. To ensure the antibody-antigen reaction and uniform distribution of PBS solution in the SU-8 reservoir, the current-voltage (I-V) measurements were initiated after 2 min from the time PBS solutions with and without antigens were introduced into the SU-8 reservoir. Figure 3 shows I-V characteristics of the Ni-salicided Si-FET device before and after adding the HA antigen solution. To directly reveal the current change caused by the specific binding of IN peptide to immobilized antibody, first the biosensor tested with control PBS solution (without peptide) was electrically measured. After the device was rinsed with deionized water and dried several times with nitrogen $\left(\mathrm{N}_{2}\right)$ gas, the biosensor was then treated with IN peptide in PBS, and the electrical measurement was performed again. The channel current of the biosensor before introducing the PBS solution into the SU-8 reservoir (the "initial" state) was extremely low $(<1 \mathrm{pA})$, as shown in the inset of Fig. 3(a). Such a low level of channel current in the initial state could be associated with a high parasitic resistance of the Si channel. Taking into account the doping concentration and geometry of the $\mathrm{Si}$ channel, the parasitic resistance was estimated to be $>10^{6} \Omega$. It is clear that the channel current of the Ni-silicided Si FET device with PBS solution alone is much higher than that in the initial state, as shown in Fig. 3(a). Abrupt increase in the channel current of the biosensor with PBS solution alone could be associated with the presence of positive ions in the PBS solution. In such a case, the electrostatic field caused by positive ions would penetrate into the Si channel, allowing the generation of majority carriers (electrons) in the $\mathrm{Si}$ channel. In other words, when compared with the initial state, the accumulation of electrons in the Si channel could be responsible for the rapid increase in the channel current. It should be noted that after specific binding of IN peptide to immobilized antibody, the channel current became significantly lower than that in the case of PBS solution alone, as shown in Fig. 3(b). A decrease in the channel current could be attributed to the negative charge of the IN peptide. Negatively charged IN peptides, introduced into the SU-8 reservoir, diffuse through the PBS solution and are selectively captured by antibodies close to the surface of the biosensor. Such specific antibody-antigen interactions lead to a build-up of electric field across the $\mathrm{SiO}_{2}$ layer and result in the generation of mobile hole carriers in a $\mathrm{Si}$ channel. In other words, the number of electrons induced in the Si channel by positive ions in the PBS solution is effectively reduced by the negatively charged peptides, which causes the significant decrease in channel current.

It should be noteworthy that $\mathrm{Ni}$-salicided $\mathrm{Si}$-FET biosensor demonstrated here is effective in the electrical immunodetection of $1.0 \mathrm{ng} / \mathrm{ml} \mathrm{IN} \mathrm{peptides} \mathrm{though} \mathrm{its} \mathrm{Si}$ channel dimension is micrometer scale (channel width $=16 \mu \mathrm{m}$ ). Nevertheless, relatively high sensing ability of Ni-salicided Si-FET biosensor could be associated with the combined effect of low doped Si channel and Ni-salicide. Generally, the sensitivity of the electrical immunodetection using FETbased biosensor fabricated by top-down process strongly depends on the dimensions and doping concentrations of the $\mathrm{Si}$ channels. For example, Kim et al. demonstrated that the decrease in channel width and doping concentration leads to the enhancement of the sensitivity of prostate-specific antigen (PSA) immunodetection using FET biosensor with p-type Si channel. ${ }^{18)}$ More precisely, the sensitivity of top-down fabricated Si channel FET biosensors is enhanced much more effectively by decreasing the doping concentration than by reducing the channel width. ${ }^{19)}$ Namely, the reduction of the doping concentration of Si channel can provide an effective way to obtain a properly high sensitivity for the electrical immunodetection of biomolecules with less effort than to reduce the channel width. However, the incorporation of Si channel with low doping concentration in FET-based biosensor results in the large increase in the channel resistance caused by source/drain underlap. This serves as a major noise source for relatively faint electrical signal produced from the channel modulation induced by the charges of biomolecules. In other word, the curtailment of source/drain underlap length using Ni-salicide process used in present device (Fig. 1) leads to the minimization of 
underlap resistance of $\mathrm{Si}$ channel, providing the effective electrical immunodetection of $1.0 \mathrm{ng} / \mathrm{ml} \mathrm{IN} \mathrm{peptides.}$

The charge of solution-based molecules is screened by dissolved solution counterions. Namely, charged species dissolved in ionic solution leads to the formation of an electrical double layer within the range of Debye length, lowering the effective charge of the biomolecules. ${ }^{20)}$ Generally, Debye length rapidly decreases with increasing the ionic concentration. Previously, experimental studies have demonstrated that for typical biological PBS solution (i.e., $1 \times$ to $0.1 \times$ PBS), the small biomolecules such as DNA or RNA oligonucelotides can be detected by means of FET-based biosensors. ${ }^{21-23)}$ On the other hand, the FET-based detection of relatively large macromolecules such as antigens through specific binding to antibody-functionalized surfaces is greatly affected by Debye screening. ${ }^{24)}$ The Debye length of PBS solution with the ionic concentration of $10 \mathrm{mM}$ used in this work is estimated to be approximately $\sim 3 \mathrm{~nm}$ when calculated assuming a simple thermal equilibrium. ${ }^{25)}$ As considering the dimensions of the anti-HA antibody, the detection of negatively charged peptides surrounded by positive ions in the PBS solution using Ni-salicided Si-FET biosensors proposed here might be infeasible since the distance between bound IN peptides and sensing channel exceeds the Debye length. ${ }^{26)}$ This could be true if intact antiHA antibodies with rigid nature are well deployed on the surface by direct adsorption, as depicted by the classical model for predicting the behavior of FET-based biosensors. ${ }^{27)}$ However, anti-HA antibodies are flexible, making it difficult to assume intact antibody. The flexible anti-HA antibodies adsorb to sensing surface in a stochastic distribution of their orientations. In other word, some anti-HA antibodies might have a configuration with orientations which would make IN peptides close enough to n-type $\mathrm{Si}$ channel. ${ }^{28)}$ Thus, the negatively charged peptides modulate electrostatic potential of n-type Si channel, leading to the decrease in channel current caused by the reduction of the number of electrons induced in the $\mathrm{Si}$ channel by positive ions in the PBS solution.

In terms of the kinetics of events taking place during the electrical transduction process in the biosensor, the target molecules (antigen-containing peptides) take a certain amount of time to diffuse toward the sensor surface and to subsequently produce a response such as an electrical signal caused by their reaction with the antibody. ${ }^{29)}$ Therefore, the time taken to reach equilibrium, at which point a stable change in signal may be determined (referred to as the "settling time"), could be the one of the major performance limitations of biosensors. ${ }^{30,31)}$ Figure 4 shows the variation in channel current, measured at a constant voltage of $2 \mathrm{~V}$, in Nisalicided Si-FET biosensors with and without antigencontaining peptide, as a function of measurement time. With increasing measurement time, the channel current with control PBS solution (no peptide) changes insignificantly. Since PBS should be nonreactive with the biosensor, it is plausible that without time delaying, numerous positive ions in the PBS solution immediately create an electrostatic field across the $\mathrm{SiO}_{2}$ layer, which in turn enhances the number of majority electron carriers in the $\mathrm{Si}$ channel. On the other hand, the biosensor treated with antigen-containing peptide

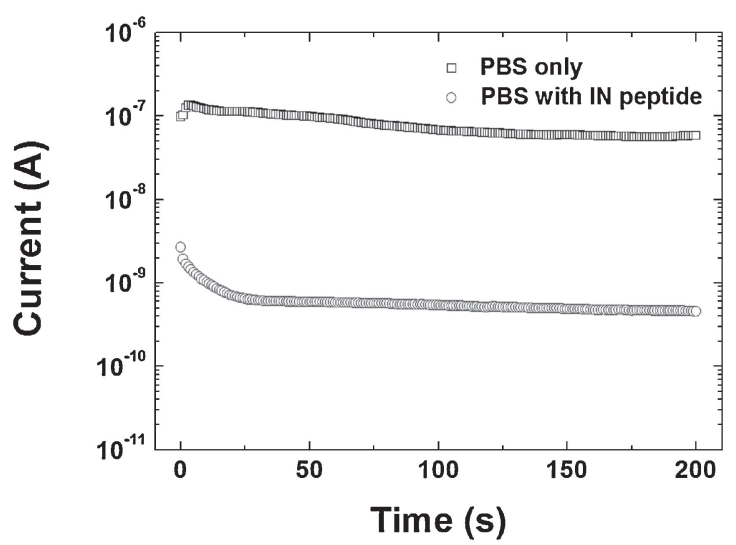

Fig. 4 Plot of variation in channel current, measured at the constant voltage of $2 \mathrm{~V}$, in Ni-salicided Si-FET biosensors with and without antigenscontaining peptide, as a function of measurement time.

in PBS undergoes a rapid decrease in channel current with increasing measurement time up to approximately $32 \mathrm{~s}$, beyond which the change in channel current is negligible. This implies that an estimate of the settling time for obtaining a stable signal change, driven by the negatively charged peptides bound to immobilized antibody, is around $32 \mathrm{~s}$.

\section{Conclusion}

We have fabricated a Si FET device with an n-type Si channel and $\mathrm{Ni}$ salicided source/drain, using a top-down process, and we have demonstrated its electrical immunodetection ability for the avian influenza A (H5N1) hemagglutinin (IN) peptide. Confocal fluorescence microscopy revealed that IN peptide was specifically bound to immobilized antibody on the patterned $\mathrm{SiO}_{2}$ overlaying the $\mathrm{Si}$ channel, confirming that the binding capability of the immobilized antibody was retained. Positively charged ions, which modulate the electrostatic field across the $\mathrm{SiO}_{2}$ layer, and which are contained in the PBS solution used in these studies, result in the accumulation of electrons in the n-type $\mathrm{Si}$ channel, leading to a rapid increase in the channel current. Upon introducing antigen-containing peptides into the SU-8 reservoir, the accumulated electrons caused by positively charged ions in the PBS solution are then reduced by the specific binding of negatively charged peptides to the immobilized antibody. From the time-dependent I-V measurements, the settling time required to stabilize the electrical signals caused by negatively charged antigens was estimated to be $32 \mathrm{~s}$.

\section{Acknowledgment}

This work was supported by the World Class University (WCU) program (R31-20029) funded by the Ministry of Education, Science, and Technology, Republic of Korea, and by the ISTD program (Grant No. 10041066) funded by the Ministry of Knowledge Economy, Republic of Korea. C-J.C. also acknowledges the support from the selection of research-oriented professor of Chonbuk National University in 2012.

H.H. and Y.-K.P. contributed equally to this work. 


\section{REFERENCES}

1) I. Capua and D. J. Alexander: Acta Trop. 83 (2002) 1-6.

2) M. D. de Jong and T. T. Hien: J. Clin. Virol. 35 (2006) 2-13.

3) A. J. Eun, L. Huang, F. T. Chew, S. F. Li and S. M. Wong: J. Virol. Methods 99 (2002) 71-79.

4) J. Xu, D. Suarez and D. S. Gottfried: Anal. Bioanal. Chem. 389 (2007) 1193-1199.

5) P. M. Boltovets, B. A. Snopok, V. R. Boyko, T. P. Shevchenko, N. S. Dyachenko and Y. M. Shirshov: J. Virol. Methods 121 (2004) 101-106.

6) K.-W. Lee, S.-J. Choi, J.-H. Ahn, D.-I. Moon, T. J. Park, S. Y. Lee and Y.-K. Choi: Appl. Phys. Lett. 96 (2010) 033703-1-033703-3.

7) T. Sakata, M. Kamahori and Y. Miyahara: Mater. Sci. Eng. C-Mater. Biol. Appl. 24 (2004) 827-832.

8) P. Wang, G. Xu, L. Qin, Y. Xu, Y. Li and R. Li: Sens. Actuator B-Chem. 108 (2005) 576-584.

9) Ch.-L. Feng, Y.-H. Xu and L.-M. Song: Sens. Actuator B-Chem. 66 (2000) 190-192.

10) M. Kamahori, Y. Ishige and M. Shimoda: Biosens. Bioelectron. 22 (2007) 3080-3085.

11) A. H. D. Graham, J. Robbins, C. R. Bowen and J. Taylor: Sensors 11 (2011) 4943-4971.

12) G. Xu, X. Ye, L. Qin, Y. Xu, Y. Li, R. Li and P. Wang: Biosens. Bioelectron. 20 (2005) 1757-1763.

13) F. Patolsky, G. Zheng, O. Hayden, M. Lakadamyali, X. Zhuang and C. M. Lieber: Proc. Natl. Acad. Sci. U.S.A. 101 (2004) 14017-14022.

14) J.-H. Ahn, S.-J. Choi, J.-W. Han, T. J. Park, S. Y. Lee and Y.-K. Choi: Nano Lett. 10 (2010) 2934-2938.

15) M. Im, J.-H. Ahn, J.-W. Han, T. J. Park, S. Y. Lee and Y.-K. Choi: IEEE Sens. J. 11 (2011) 351-360.
16) C.-J. Choi, Y.-W. Ok, S. S. Hullavarad, T.-Y. Seong, K.-M. Lee, J.-H. Lee and Y.-J. Park: J. Electrochem. Soc. 149 (2002) G517-G521.

17) C.-J. Choi, Y.-W. Ok, T.-Y. Seong and H.-D. Lee: Jpn. J. Appl. Phys. 41 (2002) 1969-1973.

18) A. Kim, C. S. Ah, H. Y. Yu, J.-H. Yang, I.-B. Baek, C.-G. Ahn, C. W. Park, M. S. Jun and S. Lee: Appl. Phys. Lett. 91 (2007) 103901-1103901-3.

19) C. W. Park, C.-G. Ahn, J.-H. Yang, I.-B. Baek, C. S. Ah, A. Kim, T.-Y. Kim and G. Y. Sung: Nanotechnology 20 (2009) 475501-1-475501-6.

20) J. N. Israelachvili: Intermolecular and Surface Forces, 2nd ed., (Academic Press, London, 1992).

21) Y. L. Bunimovich, Y. S. Shin, W.-S. Yeo, M. Amori, G. Kwong and J. R. Heath: J. Am. Chem. Soc. 128 (2006) 16323-16331.

22) Z. Li, Y. Chen, X. Li, T. I. Kamins, K. Nauka and R. S. Williams: Nano Lett. 4 (2004) 245-247.

23) G.-J. Zhang, G. Zhang, J. H. Chua, R.-E. Chee, E. H. Wong, A. Agarwal, K. D. Buddharaju, N. Singh, Z. Gao and N. Balasubramanian: Nano Lett. 8 (2008) 1066-1070.

24) P. R. Nair and M. A. Alam: IEEE Trans. Electron Devices 54 (2007) 3400-3408.

25) P. Debye: Chem. Rev. 19 (1936) 171-182.

26) P. Bergveld: Biosens. Bioelectron. 6 (1991) 55-72.

27) E. Eteshola, M. T. Keener, M. Elias, J. Shapiro, L. J. Brillson, B. Bhushan and S. C. Lee: J. R. Soc. Interf. 5 (2008) 123-127.

28) S. Gupta, M. Elias, X. Wen, J. Shapiro, L. Brillson, W. Lu and S. C. Lee: Biosens. Bioelectron. 24 (2008) 505-511.

29) A. Hassibi, S. Zahedi, R. Navid, R. W. Dutton and T. H. Lee: J. Appl. Phys. 97 (2005) 084701-1-084701-10.

30) P. R. Nair and M. A. Alam: Analyst 135 (2010) 2798-2801.

31) P. R. Nair and M. A. Alam: Nano Lett. 8 (2008) 1281-1285. 